\title{
Mathematical Study of the Small Oscillations of a Pendulum Completely Filled with a Viscoelastic Fluid
}

\author{
H. Essaouini, P. Capodanno
}

We study the small oscillations of a pendulum completely filled by a viscoelastic fluid, restricting ourselves for the fluid to the simpler Oldroyd model. We establish the equations of motion of the system. Writing them in a suitable form, we obtain an existence and unicity theorem of the solution of the associated evolution problem by means of semigroup theory. Afterwards, we show the existence and symmetry of the spectrum and prove the stability of the system. We show the existence of two sets of positive real eigenvalues, of which the first has infinity, and the second a point of the real axis, as points of accumulation. Finally, we specify the location of the possible nonreal eigenvalues.

Keywords: viscoelastic fluid, small oscillations, variational-operatorial and spectral methods, semigroups

\section{Introduction}

The mathematical study of the small oscillations of a viscous fluid in a fixed container has been the subject of numerous works which are presented in [5]. The cases of a pendulum completely and partially filled with fluid are also studied in this book.

In $[1,2]$, we have studied two problems of oscillations of a heavy viscous fluid in an immovable container. In the first work, we have considered the case of a heterogeneous fluid in an open rigid container, but in the second, we have studied the problem of a homogeneous fluid in a container closed by an elastic membrane.

Received September 25, 2019

Accepted March 30, 2020

Hilal Essaouini

hilal_essaouini@yahoo.fr

Abdelmalek Essaâdi University, Faculty of Sciences

M2SM ER28/FS/05, 93030 Tetuan, Morocco

Pierre Capodanno

pierre.capodanno@neuf .fr

Université de Franche-Comté

2B - Rue des jardins, 25000 Besançon, France

RUSSIAN JOURNAL OF NONLINEAR DYNAMICS, 2020, 16(2), 309-324 
The case of a viscoelastic fluid has been less studied. The problem of the completely or partially filled fixed container, when the fluid obeys the generalized Oldrod law, is treated in [5].

In [3], we have studied the problem of the oscillations of two nonmixing fluids, the lower inviscid, the upper viscoelastic, in an open container.

In this paper, we study the problem of the small oscillations of a pendulum that is completely filled with a viscoelastic fluid that obeys the simple Oldroyd law.

We establish the equations of motion of the pendulum-fluid system. Writing them in a suitable form, we obtain an existence and unicity theorem of the solution of the associated evolution problem by means of semigroup theory.

Afterwards, we show the existence and the symmetry of the spectrum and the stability of the system. We prove the existence of positive real eigenvalues, of which the first has infinity, and the second a point of the real axis, as points of accumulation, and give asymptotic formulae for the eigenvalues. Finally, we specify the location of the possible not real eigenvalues.

\section{The simpler Oldroyd model for a viscoelastic fluid}

It is a matter of a viscoelastic fluid for which the tensor of viscous stresses $\sigma^{\prime}$ and the doubled tensor of deformation velocities $\tau$ verify a differential equation [5]

$$
\left(1+\eta \frac{\mathrm{d}}{\mathrm{d} t}\right) \sigma^{\prime}=\left(\kappa_{0}+\kappa_{1} \frac{\mathrm{d}}{\mathrm{d} t}\right) \tau,
$$

where $\eta, \kappa_{0}, \kappa_{1}$ are some positive physical constants.

We set

$$
\frac{\kappa_{0}-\kappa_{1} \lambda}{1-\eta \lambda}=\mu\left(1+\frac{\alpha}{\gamma-\lambda}\right)
$$

with

$$
\gamma=\eta^{-1}, \quad \alpha=\frac{\kappa_{0}}{\kappa_{1}}-\gamma, \quad \mu=\kappa_{1} \gamma,
$$

which are positive constants, $\mu$ being the viscosity coefficient.

In the following, we set

$$
I_{0}(\lambda)=1+\frac{\alpha}{\gamma-\lambda}=\frac{\beta-\lambda}{\gamma-\lambda}, \quad \beta=\alpha+\gamma .
$$

We suppose [5] that, if the tensor of deformation velocities is zero at time $t=0$, the same condition is true for the tensor of viscous stresses. Under this hypothesis, we can replace the differential equation (2.1) by an integral relation

$$
\sigma^{\prime}=\mu \widehat{I}_{0}(t) \tau .
$$

Indeed, if we denote by $\Sigma^{\prime}(p)$ and $\mathcal{T}(p)$ the Laplace transforms of $\sigma^{\prime}$ and $\tau$, we have

$$
\Sigma^{\prime}(p)=\mu\left(1+\frac{\alpha}{\gamma+p}\right) \mathcal{T}(p) .
$$

From Eq. (2.5), noting that $\frac{1}{\gamma+p} \mathcal{T}(p)$ is a product of transformed, we deduce

$$
\widehat{I}_{0}(t) \tau(t)=\tau(t)+\alpha \int_{0}^{t} e^{-\gamma(t-s)} \tau(s) \mathrm{d} s .
$$




\section{Statement of the problem}

We consider a pendulum formed by a rigid $\operatorname{rod} O A$ fixed at the point $A$ to a planar rigid body $S^{\prime}$. The body $S^{\prime}+O A$ rotates about the fixed point $O$ in the plane of $S^{\prime}$. We suppose that the body $S^{\prime}+O A$ (mass $m$ ) is symmetrical with respect to $O A$ and that its center of inertia $G_{p}$ is on $O A$.

The body $S^{\prime}$ has a cavity symmetrical with respect to $O A$, completely filled with a homogeneous incompressible viscoelastic fluid (mass $M$, density $\rho$ ), the center of inertia of which $G_{f}$ is on $O A$.

In the equilibrium position, $O A$ is directed vertically downwards. $\Omega$ and $S$ are, respectively, the domain occupied by the fluid and the wall of the cavity.

We use a fixed orthonormal axes $O x_{1} x_{2}, O x_{2}$ vertically directed upwards and orthonormal axes $O X_{1} X_{2}$, fixed in the pendulum [Fig. 1]; we set $O \widehat{x_{1}, O X_{1}}=\theta ; \theta$ and its derivatives are supposed to be small.

We denote by $-g \vec{x}_{2}$ the constant acceleration of gravity. We are going to study the small oscillations of the pendulum-fluid system in linear theory.

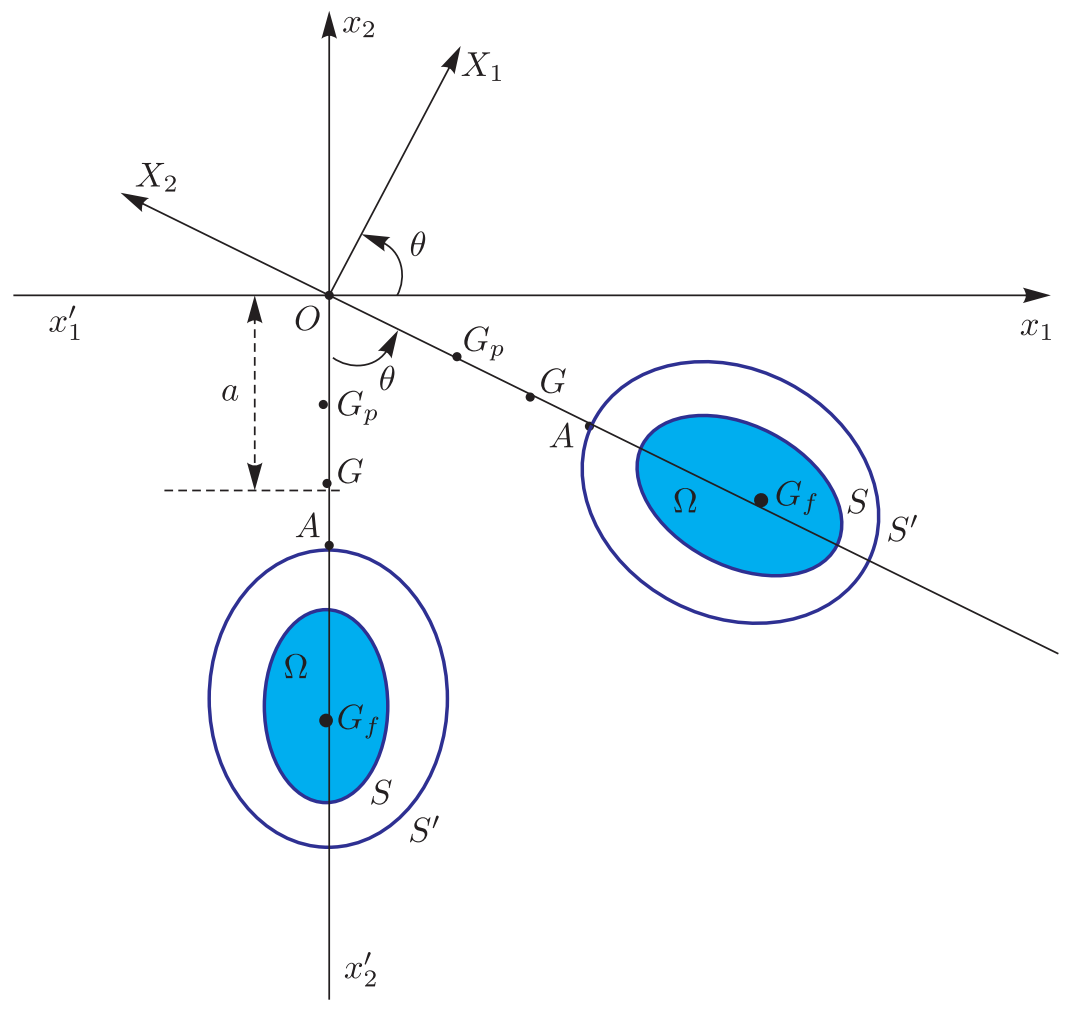

Fig. 1. Model of the system.

\section{Equations of motion of the system}

We denote by $\vec{u}\left(x_{1}, x_{2}, t\right)$ the (small) displacement of a fluid particle $M$ with respect to the axes $O X_{1} X_{2}$; its displacement with respect to the fixed axes $O x_{1} x_{2}$ is

$$
\vec{U}=\vec{u}+\theta \vec{z} \times \overrightarrow{O M} \quad(\vec{z}=\vec{x} \times \vec{y}) .
$$




\subsection{Application of the theorem of moment of inertia to the body-fluid system}

If $G$ is its center of inertia $\left(\overrightarrow{O G}=-a \vec{X}_{2} ;(m+M) \overrightarrow{O G}=m \overrightarrow{O G}_{p}+M \overrightarrow{O G}_{f}\right)$ and $J_{p}$ the moment of inertia with respect to $O$ of the pendulum, we have

$$
J_{p} \ddot{\theta} \vec{z}+\int_{\Omega} \rho \overrightarrow{O M} \times \ddot{\vec{U}} \mathrm{~d} \Omega=\overrightarrow{O G} \times\left[-(m+M) g \vec{x}_{2}\right]
$$

or, using (4.1),

$$
J_{p} \ddot{\theta}+\rho \int_{\Omega}\left[x_{1}\left(\ddot{u}_{2}+\ddot{\theta} x_{1}\right)-x_{2}\left(\ddot{u}_{1}-\ddot{\theta} x_{2}\right)\right] \mathrm{d} \Omega=-(m+M) g a \theta,
$$

and, denoting by $J$ the moment of inertia in $O$ of the pendulum-fluid

$$
J \ddot{\theta}+\rho \int_{\Omega}\left(x_{1} \ddot{u}_{2}-x_{2} \ddot{u}_{1}\right) \mathrm{d} \Omega=-(m+M) g a \theta .
$$

\subsection{Equations of motion of the viscoelastic fluid}

If $P\left(x_{1}, x_{2}, t\right)$ is the pressure, we have

$$
\begin{aligned}
& \rho \ddot{U}_{i}=-\frac{\partial P}{\partial x_{i}}+\frac{\partial \sigma_{i j}^{\prime}}{\partial x_{j}}-\rho g \delta_{i 3} \quad \text { in } \quad \Omega \quad(i, j=1,2,3), \\
& \operatorname{div} \vec{u}=0 \quad \text { in } \quad \Omega, \\
& \vec{u}_{\mid S}=0 .
\end{aligned}
$$

If we denote by

$$
\tau_{i j}=\frac{\partial \dot{u}_{i}}{\partial x_{j}}+\frac{\partial \dot{u}_{j}}{\partial x_{i}} \quad(i, j=1,2,3)
$$

the components of the tensor $\tau$, we have for the tensor $\sigma^{\prime}$

$$
\sigma_{i j}^{\prime}=\mu \widehat{I}_{0} \tau_{i j}
$$

and then

$$
\frac{\partial \sigma_{i j}^{\prime}}{\partial x_{j}}=\mu \widehat{I}_{0} \frac{\partial \tau_{i j}}{\partial x_{j}}=\mu \widehat{I}_{0}\left(\frac{\partial^{2} \dot{u}_{i}}{\partial x_{j} \partial x_{j}}+\frac{\partial}{\partial x_{i}}\left(\frac{\partial \dot{u}_{j}}{\partial x_{j}}\right)\right)=\mu \widehat{I}_{0} \Delta \dot{u}_{i}
$$

Equation (4.3) takes the form

$$
\rho \ddot{\vec{U}}=-\overrightarrow{\operatorname{grad}} P+\mu \widehat{I_{0}} \Delta \dot{\vec{U}}-\rho g \vec{x}_{2} .
$$

The pressure in the equilibrium position is

$$
P_{s t}=-\rho g x_{2}+\text { const. }
$$

If we introduce the dynamic pressure $p=P-P_{s t}$, equation (4.6) can be written as

$$
\rho \ddot{\vec{u}}=-\overrightarrow{\operatorname{grad}} p+\mu \widehat{I_{0}} \Delta \dot{\vec{u}}-\rho \ddot{\theta} \vec{z} \times \overrightarrow{O M} .
$$

Equations (4.2), (4.7), (4.4), (4.5) are the equations of the problem.

REMARK. If the fluid is solidified $(\vec{u}=0)$, we have the classical pendulum and Eq. (4.2) gives its pulsation $\Lambda$ by the formula

$$
\Lambda^{2}=\frac{(m+M) g a}{J} .
$$




\section{The operatorial equations of the problem}

\subsection{A few transformations}

We seek $\theta$ in $\mathbb{C}$ and $\vec{u}$ in the space [4]

$$
J_{0}^{1}(\Omega)=\left\{\vec{u} \in \chi^{1}(\Omega) \stackrel{\text { def }}{=}\left[H^{1}(\Omega)\right]^{2}, \quad \operatorname{div} \vec{u}=0 \quad \text { in } \Omega, \quad \vec{u}_{\mid S}=0\right\}
$$

equipped with the scalar product

$$
(\vec{u}, \overrightarrow{\tilde{u}})_{J_{0}^{1}(\Omega)}=2 \int_{\Omega} \epsilon_{i j}(\vec{u}) \epsilon_{i j}(\overline{\overrightarrow{\tilde{u}}}) \mathrm{d} \Omega .
$$

It is well known that the associated norm is equivalent in this space to the classical norm of $\chi^{1}(\Omega)$.

We introduce the space [4]

$$
J_{0}(\Omega)=\left\{\vec{u} \in \mathscr{L}^{2}(\Omega) \stackrel{\text { def }}{=}\left[L^{2}(\Omega)\right]^{2}, \operatorname{div} \vec{u}=0, u_{n \mid S}=0\right\}
$$

equipped with the norm of $\mathscr{L}^{2}(\Omega)$.

It is well known [4] that the embedding from $J_{0}^{1}(\Omega) \subset J_{0}(\Omega)$ is continuous, dense and compact and we denote by $A$ the unbounded operator of $J_{0}(\Omega)$ associated to the pair $\left(J_{0}^{1}(\Omega), J_{0}(\Omega)\right)$ and to the scalar product of $J_{0}^{1}(\Omega)$.

Introducing $\overrightarrow{\widetilde{u}}\left(x_{1}, x_{2}\right) \in J_{0}^{1}(\Omega)$, we have, using (4.7),

$$
\int_{\Omega} \rho \ddot{\vec{u}} \cdot \overline{\vec{u}} \mathrm{~d} \Omega=-\int_{\Omega} \overrightarrow{\operatorname{grad}} p \cdot \overline{\overrightarrow{\vec{u}}} \mathrm{~d} \Omega+\mu \int_{\Omega} \widehat{I_{0}} \Delta \dot{\vec{u}} \cdot \overline{\vec{u}} \mathrm{~d} \Omega-\rho \ddot{\theta} \int_{\Omega}(\vec{z}, \overrightarrow{\mathrm{OM}}, \overrightarrow{\vec{u}}) \mathrm{d} \Omega .
$$

Using the Green formula, we have

$$
\begin{aligned}
\int_{\Omega} \overrightarrow{\operatorname{grad}} p \cdot \overline{\overrightarrow{\vec{u}}} \mathrm{~d} \Omega & =\int_{\Omega}[\operatorname{div}(p \overline{\overrightarrow{\vec{u}}})-p \operatorname{div} \overline{\overrightarrow{\vec{u}}}] \mathrm{d} \Omega \\
& =\int_{S} p \overline{\widetilde{u}}_{n \mid S} \mathrm{~d} S \\
& =0 .
\end{aligned}
$$

On the other hand, with $\widehat{I}_{0}$ permuting obviously with $\Delta$, we have

$$
\mu \int_{\Omega} \widehat{I}_{0} \Delta \dot{\vec{u}} \cdot \overline{\vec{u}} \mathrm{~d} \Omega=\mu \widehat{I}_{0} \int_{\Omega} \Delta \dot{\vec{u}} \cdot \overline{\vec{u}} \mathrm{~d} \Omega .
$$

The formula of the vectorial Laplacian [4] gives

$$
\begin{aligned}
\int_{\Omega} \Delta \dot{\vec{u}} \cdot \overline{\vec{u}} \mathrm{~d} \Omega & =-2 \int_{\Omega} \epsilon_{i j}(\dot{\vec{u}}) \epsilon_{i j}(\overline{\vec{u}}) \mathrm{d} \Omega+2 \int_{S} \epsilon_{i j}(\dot{\vec{u}}) \overline{\widetilde{u}}_{j} n_{i} \mathrm{~d} S \\
& =-2 \int_{\Omega} \epsilon_{i j}(\dot{\vec{u}}) \epsilon_{i j}(\overline{\overrightarrow{\widetilde{u}}}) \mathrm{d} \Omega .
\end{aligned}
$$


Finally, we have

$$
\int_{\Omega}(\vec{z}, \overrightarrow{\mathrm{OM}}, \overrightarrow{\widetilde{u}}) \mathrm{d} \Omega=\left(x_{1} \vec{x}_{2}-x_{2} \vec{x}_{1}, \overrightarrow{\widetilde{u}}\right)_{\mathscr{L}^{2}(\Omega)}=\left(\mathcal{P}_{0}\left(x_{1} \vec{x}_{2}-x_{2} \vec{x}_{1}\right), \overrightarrow{\widetilde{u}}\right)_{\mathscr{L}^{2}(\Omega)}
$$

denoting by $\mathcal{P}_{0}$ the orthogonal projector of $\mathscr{L}^{2}(\Omega)$ into $J_{0}(\Omega)$.

We obtain the variational equation

$$
(\ddot{\vec{u}}, \overrightarrow{\widetilde{u}})_{\mathscr{L}^{2}(\Omega}+2 \mu \widehat{I}_{0} \int_{\Omega} \epsilon_{i j}(\dot{\vec{u}}) \epsilon_{i j}(\overline{\overrightarrow{\widetilde{u}}}) \mathrm{d} \Omega+\rho\left(\mathcal{P}_{0}\left(x_{1} \vec{x}_{2}-x_{2} \vec{x}_{1}\right) \ddot{\theta}, \overrightarrow{\widetilde{u}}\right)_{\mathscr{L}^{2}(\Omega)}=0 \quad \forall \overrightarrow{\widetilde{u}} \in J_{0}^{1}(\Omega) .
$$

It is well known [6] that this equation is equivalent to the operatorial equation

$$
\ddot{\vec{u}}+\nu \widehat{I}_{0} A \dot{\vec{u}}+\mathcal{P}_{0}\left(x_{1} \vec{x}_{2}-x_{2} \vec{x}_{1}\right) \ddot{\theta}=0,
$$

where we have set

$$
\nu=\frac{\mu}{\rho} .
$$

Let us introduce the operator $L$ from $J_{0}(\Omega)$ into $\mathbb{C}$ defined by

$$
L \vec{u}=\int_{\Omega}\left(x_{1} u_{2}-x_{2} u_{1}\right) \mathrm{d} \Omega .
$$

Equation (4.2) takes the form

$$
\frac{J}{\rho} \ddot{\theta}+L \ddot{\vec{u}}+\frac{(m+M) g a}{\rho} \theta=0 .
$$

It is easy to see that $L$ and $\mathcal{P}_{0}\left(x_{1} \vec{x}_{2}-x_{2} \vec{x}_{1}\right)$ are mutually adjoint.

\subsection{New forms of Eqs. (5.1) and (5.2)}

We are going to prove that we can reduce Eqs. (5.1) and (5.2) to a system with four differential equations of the first order with constant coefficients:

Setting

$$
\vec{v}=\dot{\vec{u}}, \quad \omega=\dot{\theta},
$$

we obtain

$$
\left\{\begin{array}{l}
\dot{\vec{v}}+\mathcal{P}_{0}\left(x_{1} \vec{x}_{2}-x_{2} \vec{x}_{1}\right) \dot{\omega}+\nu \widehat{I}_{0} A \vec{v}=0 \\
L \dot{\vec{v}}+\frac{J}{\rho} \dot{\omega}+\frac{(m+M) g a}{\rho} \theta=0 \\
\dot{\theta}=\omega .
\end{array}\right.
$$

We have

$$
\widehat{I_{0}} A \vec{v}=A \vec{v}(x, t)+\alpha \int_{0}^{t} e^{-\gamma(t-s)} A \vec{v}(x, s) \mathrm{d} s .
$$

If we set

$$
\vec{v}_{1}=(\nu \alpha)^{1 / 2} \int_{0}^{t} e^{-\gamma(t-s)} A^{1 / 2} \vec{v}(x, s) \mathrm{d} s,
$$

we obtain

$$
\widehat{I}_{0} A \vec{v}=A \vec{v}+\alpha(\nu \alpha)^{-1 / 2} A^{1 / 2} \vec{v}_{1}
$$


and finally

$$
\dot{\vec{v}}+\mathcal{P}_{0}\left(x_{1} \vec{x}_{2}-x_{2} \vec{x}_{1}\right) \dot{\omega}+\nu A \vec{v}+(\nu \alpha)^{1 / 2} A^{1 / 2} \vec{v}_{1}=0 .
$$

On the other hand, we have

$$
\dot{\vec{v}}_{1}=(\nu \alpha)^{1 / 2}\left[\int_{0}^{t}-\gamma e^{-\gamma(t-s)} A^{1 / 2} \vec{v}(x, s) \mathrm{d} s+A^{1 / 2} \vec{v}(x, t)\right]
$$

or

$$
\dot{\vec{v}}_{1}=-\gamma \vec{v}_{1}+(\nu \alpha)^{1 / 2} A^{1 / 2} \vec{v} .
$$

Consequently, we can replace Eqs. (5.1) and (5.2) by the system of four differential equations:

$$
\begin{aligned}
& \dot{\vec{v}}+\mathcal{P}_{0}\left(x_{1} \vec{x}_{2}-x_{2} \vec{x}_{1}\right) \dot{\omega}+\nu A \vec{v}+(\nu \alpha)^{1 / 2} A^{1 / 2} \vec{v}_{1}=0, \\
& \dot{\vec{v}}+\gamma \vec{v}_{1}-(\nu \alpha)^{1 / 2} A^{1 / 2} \vec{v}=0, \\
& L \dot{\vec{v}}+\frac{J}{\rho} \dot{\omega}+\frac{(m+M) g a}{\rho} \theta=0, \\
& \frac{(m+M) g a}{\rho} \dot{\theta}-\frac{(m+M) g a}{\rho} \omega=0 .
\end{aligned}
$$

\subsection{Operatorial equation of the problem}

Let us assume that

$$
z=\left(\begin{array}{c}
\vec{v} \\
\vec{v}_{1} \\
\omega \\
\theta
\end{array}\right) \in \chi \stackrel{\text { def }}{=} J_{0}(\Omega) \oplus J_{0}(\Omega) \oplus \mathbb{C} \oplus \mathbb{C} .
$$

Then we can write the precedent equations in the form

$$
\mathcal{A}=\left(\begin{array}{cccc}
I_{J_{0}(\Omega)} & 0 & \mathcal{P}_{0}\left(x_{1} \vec{x}_{2}-x_{2} \vec{x}_{1}\right) & 0 \\
0 & I_{J_{0}(\Omega)} & 0 & 0 \\
L & 0 & \frac{J}{\rho} I_{\mathbb{C}} & 0 \\
0 & 0 & 0 & \frac{(m+M) g a}{\rho} I_{\mathbb{C}}
\end{array}\right)
$$

with

$$
\mathcal{B}=\left(\begin{array}{cccc}
\nu A & (\nu \alpha)^{1 / 2} A^{1 / 2} & 0 & 0 \\
-(\nu \alpha)^{1 / 2} A^{1 / 2} & \gamma I_{J_{0}(\Omega)} & 0 & 0 \\
0 & 0 & 0 & \frac{(m+M) g a}{\rho} I_{\mathbb{C}} \\
0 & 0 & -\frac{(m+M) g a}{\rho} I_{\mathbb{C}} & 0
\end{array}\right)
$$

where $\mathcal{A}$ is a bounded operator from $\chi$ into $\chi, \mathcal{B}$ is an unbounded operator on $\chi$, and its domain is

$$
\mathcal{D}(\mathcal{B})=\mathcal{D}(\mathcal{A}) \oplus \mathcal{D}\left(\mathcal{A}^{1 / 2}\right) \oplus \mathbb{C} \oplus \mathbb{C} .
$$




\subsection{Properties of the operators $\mathcal{A}$ and $\mathcal{B}$}

\subsubsection{Properties of the operator $\mathcal{A}$}

$\mathcal{A}$ is self-adjoint and strongly positive.

Indeed, we have easily

$$
\left\{\begin{aligned}
(\mathcal{A} z, z)_{\chi} & =\|\vec{v}\|_{J_{0}(\Omega)}^{2}+\left\|\vec{v}_{1}\right\|_{J_{0}(\Omega)}^{2}+\frac{J}{\rho}|\omega|^{2}+\frac{(m+M) g a}{\rho}|\theta|^{2} \\
& +2 \operatorname{Re}\left(\mathcal{P}_{0}\left(x_{1} \vec{x}_{2}-x_{2} \vec{x}_{1}\right) \omega, \vec{v}\right)_{J_{0}(\Omega)} .
\end{aligned}\right.
$$

But we have

$$
\left\|\mathcal{P}_{0}\left(x_{1} \vec{x}_{2}-x_{2} \vec{x}_{1}\right) \omega\right\|_{J_{0}(\Omega)} \leqslant\left(\int_{\Omega}\left(x_{1}^{2}+x_{2}^{2}\right) \mathrm{d} \Omega\right)^{1 / 2}|\omega|=\sqrt{\frac{J_{\ell}}{\rho}}|\omega|,
$$

where $J_{\ell}=J-J_{p}$ is the moment of inertia of the fluid about $O$.

Consequently, we have

$$
\left\{\begin{aligned}
(\mathcal{A} z, z)_{\chi} & \geqslant\left\|\vec{v}_{1}\right\|_{J_{0}(\Omega)}^{2}+\frac{(m+M) g a}{\rho}|\theta|^{2} \\
& +\left[\|\vec{v}\|_{J_{0}(\Omega)}^{2}+\frac{J}{\rho}|\omega|^{2}-2 \sqrt{\frac{J_{\ell}}{\rho}}|\omega|\|\vec{v}\|_{J_{0}(\Omega)}\right] .
\end{aligned}\right.
$$

The quadratic form between the brackets is positive definite because $J \geqslant J_{\ell}$.

Finally, we have

$$
(\mathcal{A} z, z)_{\chi} \geqslant C\|z\|_{\chi}^{2}, \quad C>0
$$

\subsubsection{Properties of the operator $\mathcal{B}$}

i) $\mathcal{B}$ is not self-adjoint, but it has a bounded inverse.

Indeed, let

$$
\widetilde{z}=\left(\overrightarrow{\vec{v}}, \overrightarrow{\widetilde{v}}_{1}, \widetilde{\omega}, \widetilde{\theta}\right)^{t} \in \chi
$$

We are going to solve the equation

$$
\mathcal{B} z=\widetilde{z}, \quad z \in \mathcal{D}(\mathcal{B})
$$

i.e.,

$$
\left\{\begin{array} { l } 
{ \nu A \vec { v } + ( \nu \alpha ) ^ { 1 / 2 } A ^ { 1 / 2 } \vec { v } _ { 1 } = \vec { \widetilde { v } } , } \\
{ - ( \nu \alpha ) ^ { 1 / 2 } A ^ { 1 / 2 } \vec { v } + \gamma \vec { v } _ { 1 } + = \vec { \widetilde { v } } _ { 1 } , }
\end{array} \quad \left\{\begin{array}{l}
\frac{(m+M) g a}{\rho} \theta=\widetilde{\omega}, \\
-\frac{(m+M) g a}{\rho} \omega=\widetilde{\theta} .
\end{array}\right.\right.
$$

We have

$$
\theta=\frac{\rho}{(m+M) g a} \widetilde{\omega}, \quad \omega=-\frac{\rho}{(m+M) g a} \widetilde{\theta},
$$


and hence

$$
\left\{\begin{array}{l}
\vec{v}_{1}=\frac{1}{\gamma}\left(\overrightarrow{\vec{v}}_{1}+(\nu \alpha)^{1 / 2} A^{1 / 2} \vec{v}\right) \\
\vec{v}=\frac{\gamma}{\nu \beta} A^{-1} \overrightarrow{\vec{v}}-\frac{(\nu \alpha)^{1 / 2}}{\nu \beta} A^{-1 / 2} \overrightarrow{\widetilde{v}}_{1} \\
\vec{v}_{1}=\frac{(\nu \alpha)^{1 / 2}}{\nu \beta} A^{-1 / 2} \overrightarrow{\widetilde{v}}+\frac{1}{\beta} \overrightarrow{\widetilde{v}}_{1}
\end{array}\right.
$$

Then $\mathcal{B}^{-1}$ exists and we have

$$
\mathcal{B}^{-1}=\left(\begin{array}{cccc}
\frac{\gamma}{\nu \beta} A^{-1} & -\frac{(\nu \alpha)^{1 / 2}}{\nu \beta} A^{-1 / 2} & 0 & 0 \\
\frac{(\nu \alpha)^{1 / 2}}{\nu \beta} A^{-1 / 2} & \frac{1}{\beta} I_{J_{0}(\Omega)} & 0 & 0 \\
0 & 0 & 0 & -\frac{\rho}{(m+M) g a} I_{\mathbb{C}} \\
0 & 0 & \frac{\rho}{(m+M) g a} I_{\mathbb{C}} & 0
\end{array}\right)
$$

where $\mathcal{B}^{-1}$ is not self-adjoint and not compact.

ii) $\mathcal{B}$ has a remarkable property: $-\mathcal{B}$ is maximal dissipative.

Indeed, we have easily

$$
\left\{\begin{array}{l}
(\mathcal{B} z, z)_{\chi}=\nu(A \vec{v}, \vec{v})_{J_{0}(\Omega)}+\gamma\left\|\vec{v}_{1}\right\|_{J_{0}(\Omega)}^{2} \\
+(\nu \alpha)^{1 / 2}\left[\left(A^{1 / 2} \vec{v}_{1}, \vec{v}\right)_{J_{0}(\Omega)}-\left(A^{1 / 2} \vec{v}, \vec{v}_{1}\right)_{J_{0}(\Omega)}\right]+\frac{(m+M) g a}{\rho}(\theta \bar{\omega}-\omega \bar{\theta})
\end{array}\right.
$$

so that

$$
\operatorname{Re}(\mathcal{B} z, z)_{\chi}=\nu(A \vec{v}, \vec{v})_{J_{0}(\Omega)}+\gamma\left\|\vec{v}_{1}\right\|_{J_{0}(\Omega)}^{2} \geqslant 0 .
$$

On the other hand, we have

$$
\mathcal{B}^{*}=\left(\begin{array}{cccc}
\nu A & -(\nu \alpha)^{1 / 2} A^{1 / 2} & 0 & 0 \\
(\nu \alpha)^{1 / 2} A^{1 / 2} & \gamma I_{J_{0}(\Omega)} & 0 & 0 \\
0 & 0 & 0 & -\frac{(m+M) g a}{\rho} I_{\mathbb{C}} \\
0 & 0 & \frac{(m+M) g a}{\rho} I_{\mathbb{C}} & 0
\end{array}\right)
$$

so that we have in the same manner

$$
\operatorname{Re}\left(\mathcal{B}^{*} z, z\right)_{\chi} \geqslant 0
$$

and $-\mathcal{B}$ is maximal dissipative $[4,7]$. 


\section{Existence and unicity of the solution of the associated evolution problem}

Since $\mathcal{A}$ is bounded, self-adjoint and strongly positive, we can set

$$
\mathcal{A}^{1 / 2} z=\zeta \in \chi
$$

and Eq. (5.7) becomes

$$
\dot{\zeta}+\mathcal{A}^{-1 / 2} \mathcal{B A}^{-1 / 2} \zeta=0
$$

At first, we have

$$
\operatorname{Re}\left(\mathcal{A}^{-1 / 2} \mathcal{B A}^{-1 / 2} \zeta, \zeta\right)_{\chi}=\operatorname{Re}(\mathcal{B} z, z)_{\chi} \geqslant 0
$$

On the other hand, since $\mathcal{A}^{-1 / 2}$ is bounded and self-adjoint, we have

$$
\left(\mathcal{A}^{-1 / 2} \mathcal{B A}^{-1 / 2}\right)^{*}=\mathcal{A}^{-1 / 2} \mathcal{B}^{*} \mathcal{A}^{-1 / 2}
$$

and

$$
\operatorname{Re}\left(\mathcal{A}^{-1 / 2} \mathcal{B}^{*} \mathcal{A}^{-1 / 2} \zeta, \zeta\right)_{\chi}=\operatorname{Re}\left(\mathcal{B}^{*} z, z\right)_{\chi} \geqslant 0
$$

so $-\mathcal{A}^{-1 / 2} \mathcal{B} \mathcal{A}^{-1 / 2}$ is maximal dissipative.

Consequently, $[4,7]$, if $z(0) \in \mathcal{D}(\mathcal{A})$, i.e, if

$$
\vec{v}(0) \in \mathcal{D}(A), \quad \vec{v}_{1}(0) \in \mathcal{D}\left(A^{1 / 2}\right),
$$

the problem (5.7) has one and only one solution belonging to $\mathcal{D}(\mathcal{A})$ for $t \geqslant 0$.

\section{Normal oscillations; first results about the spectrum}

\subsection{Normal oscillations}

Setting

$$
z\left(x_{1}, x_{2}, t\right)=e^{-\lambda t} z\left(x_{1}, x_{2}\right), \quad \lambda \in \mathbb{C},
$$

we have

$$
-\lambda \mathcal{A} z+\mathcal{B} z=0
$$

or

$$
\begin{aligned}
& -\lambda\left(\overrightarrow{v+\mathcal{P}_{0}}\left(x_{1} \vec{x}_{2}-x_{2} \vec{x}_{1}\right) \omega\right)+\nu A \vec{v}+(\nu \alpha)^{1 / 2} A^{1 / 2} \vec{v}_{1}=0, \\
& -\lambda \vec{v}_{1}-(\nu \alpha)^{1 / 2} A^{1 / 2} \vec{v}+\gamma \vec{v}_{1}=0, \\
& -\lambda\left(L \vec{v}+\frac{J}{\rho} \omega\right)+\frac{(m+M) g a}{\rho} \theta=0, \\
& -\lambda \theta-\omega=0 .
\end{aligned}
$$

7.2. $\lambda=0$ is not an eigenvalue

Indeed, if $z$ is an eigenelement associated to the eigenvalue $\lambda=0$, we have $\mathcal{B} z=0$ and then $z=0$ since $\mathcal{B}$ has an inverse. 


\section{3. $\lambda=\gamma$ is not an eigenvalue}

Indeed, if $\lambda=\gamma$ is an eigenvalue, (7.2) gives $\vec{v}=0$; then (7.3) and (7.4) give $\theta=0, \omega=0$ and finally $(7.2)$ gives $\vec{v}_{1}=0$.

\subsection{The spectrum is in the half-plane $\operatorname{Re} \lambda>0$}

Indeed, if $\lambda$ is an eigenvalue and $z$ a corresponding eigenelement, we have

$$
\lambda(\mathcal{A} z, z)_{\chi}=(\mathcal{B} z, z)_{\chi}
$$

Taking the real parts, we have, $(\mathcal{A} z, z)_{\chi}$ being real and positive,

$$
\operatorname{Re} \lambda(\mathcal{A} z, z)_{\chi}=\operatorname{Re}(\mathcal{B} z, z)_{\chi}=\nu(A \vec{v}, \vec{v})_{J_{0}(\Omega)}+\gamma\left\|\vec{v}_{1}\right\|_{J_{0}(\Omega)}^{2},
$$

so that

$$
\operatorname{Re} \lambda \geqslant 0
$$

Let us prove that $\operatorname{Re} \lambda=0$ is impossible.

By virtue of the precedent result, if $\operatorname{Re} \lambda=0$, we have

$$
\vec{v}=0, \quad \vec{v}_{1}=0
$$

and consequently

$$
\left\{\begin{array}{l}
-\lambda \mathcal{P}_{0}\left(x_{1} \vec{x}_{2}-x_{2} \vec{x}_{1}\right) \omega=0 \\
-\lambda \frac{J}{\rho} \omega+\frac{(m+M) g a}{\rho} \theta=0 \\
-\lambda \theta-\omega=0
\end{array}\right.
$$

We have

$$
\left(\lambda^{2}+\Lambda^{2}\right) \theta=0
$$

and $\lambda \neq \pm i \Lambda$ cannot be an eigenvalue.

Let us study the case $\lambda= \pm i \Lambda$. Then we have

$$
\mathcal{P}_{0}\left(x_{1} \vec{x}_{2}-x_{2} \vec{x}_{1}\right) \omega=0, \quad \pm i \Lambda \theta-\omega=0 .
$$

The first equation proves that $\left(x_{1} \vec{x}_{2}-x_{2} \vec{x}_{1}\right) \omega$ belongs to the space $\mathscr{G}(\Omega)$ of the potential fields, i.e., it has the form $\overrightarrow{\operatorname{grad}} \varphi$; therefore, we have

$$
\frac{\partial \varphi}{\partial x_{1}}=-\omega x_{2}, \quad \frac{\partial \varphi}{\partial x_{2}}=\omega x_{1}
$$

It is possible only if $\omega=0$ and then $\theta=0$.

Finally, the spectrum lies in the half-plane $\operatorname{Re} \lambda>0$ and the system is linearly stable. 


\subsection{The existence and the symmetry of the spectrum}

From (7.3) and (7.4), we deduce

$$
\omega=F(\lambda)^{-1} L \vec{v}, \quad \text { with } \quad F(\lambda)=\frac{\frac{J}{\rho} \lambda^{2}+\frac{(m+M) g a}{\rho}}{\lambda^{2}} .
$$

Since $\lambda=\gamma$ is not an eigenvalue, we can, to study the spectrum, divide Eq. (7.2) by $\lambda-\gamma$ :

$$
\vec{v}_{1}=-\frac{(\nu \alpha)^{1 / 2}}{\lambda-\gamma} A^{1 / 2} \vec{v}
$$

Therefore, Eq. (7.1) becomes

$$
-\lambda \vec{v}-\lambda F(\lambda)^{-1} \mathcal{P}_{0}\left(x_{1} \vec{x}_{2}-x_{2} \vec{x}_{1}\right) L \vec{v}+\nu I_{0}(\lambda) A \vec{v}=0,
$$

where we have set

$$
I_{0}(\lambda)=\frac{\lambda-\beta}{\lambda-\gamma}
$$

Setting $A^{1 / 2} \vec{v}=\vec{w} \in J_{0}(\Omega)$ and applying the operator $A^{-1 / 2}$, we obtain the equation with bounded coefficients

$$
\mathscr{L}(\lambda) \vec{w} \stackrel{\text { def }}{=}-\lambda A^{-1} \vec{w}-\lambda F(\lambda)^{-1} B \vec{w}+\nu I_{0}(\lambda) \vec{w}=0,
$$

where we have set

$$
B=A^{-1 / 2} \mathcal{P}_{0}\left(x_{1} \vec{x}_{2}-x_{2} \vec{x}_{1}\right) L A^{-1 / 2} .
$$

$B$ is an operator from $J_{0}(\Omega)$ into $J_{0}(\Omega)$, self-adjoint, compact; it is nonnegative, since

$$
(B \vec{w}, \vec{w})_{J_{0}(\Omega)}=\|L \vec{v}\|_{\mathbb{C}}^{2} \geqslant 0 .
$$

Let us show that $\lambda=\beta$ is not an eigenvalue.

Otherwise, if $\vec{w}$ were a corresponding eigenvector, we would have

$$
\beta A^{-1} \vec{w}+\beta F(\beta)^{-1} B \vec{w}=0,
$$

and then

$$
\left(A^{-1} \vec{w}, \vec{w}\right)_{J_{0}(\Omega)}+\beta F(\beta)^{-1}(B \vec{w}, \vec{w})_{J_{0}(\Omega)}=0 .
$$

But it is obviously impossible, the left-hand side being strictly positive.

Therefore, we can devide Eq. (7.5) and obtain

$$
\mathscr{L}_{0}(\lambda) \vec{w} \stackrel{\text { def }}{=}\left(I_{J_{0}(\Omega)}-\frac{\lambda}{\nu I_{0}(\lambda)} A^{-1}-\frac{\lambda}{\nu F(\lambda) I_{0}(\lambda)} B\right) \vec{w}=0,
$$

where $\mathscr{L}_{0}(\lambda)$ is a holomorphic operatorial functional in the domain $\mathbb{C}-\{\lambda= \pm i \Lambda\}-\{\lambda=\beta\}$; on the other hand, $A^{-1}$ and $B$ are compact.

Therefore, the pencil $\mathscr{L}_{0}(\lambda)$ is a Fredholm pencil in the domain. It is regular, because $\lambda=\gamma$ is not an eigenvalue.

Then, according to [4], the spectrum of the pencil exists and is formed by isolated points in the domain. All the points of the spectrum are eigenvalues with possible points of accumulation $\lambda=\beta, \lambda= \pm i \Lambda, \lambda=\infty$. The eigenelements have finite multiplicity.

Finally, $A^{-1}$ and $B$ being selfadjoint, the pencil $\mathscr{L}_{0}(\lambda)$ is selfadjoint and its spectrum is symmetrical with respect to the real axis.

In the following, we are going to prove a few properties of the spectrum. 


\section{There are not real eigenvalue in the closed interval $[\gamma, \beta]$}

We know that $\gamma$ is not an eigenvalue. We are going to prove that $\lambda=\beta-\varepsilon$ with $0 \leqslant \varepsilon<0$ cannot be an eigenvalue.

We can consider the pencil

$$
\mathcal{M}(\lambda)=(\gamma-\lambda) \mathscr{L}(\lambda)
$$

and we have

$$
\mathcal{M}(\beta-\varepsilon)=\nu \varepsilon I_{J_{0}(\Omega)}+(\beta-\varepsilon)(\beta-\gamma-\varepsilon) A^{-1}-\frac{\rho}{J} \frac{(\beta-\varepsilon)^{3}(\beta+\gamma-\varepsilon)}{(\beta-\varepsilon)^{2}+\Lambda^{2}} .
$$

If $\beta-\varepsilon$ is an eigenvalue, there exists a normalized element $\vec{w} \in J_{0}(\Omega)\left(\|\vec{w}\|_{J_{0}(\Omega)}=1\right)$ such that $\mathcal{M}(\beta-\varepsilon) \vec{w}=0$. Let us show that it is impossible.

We set

$$
p=\left(A^{-1} \vec{w}, \vec{w}\right)_{J_{0}(\Omega)}, \quad q=(B \vec{w}, \vec{w})_{J_{0}(\Omega)} .
$$

We have found

$$
p=\|\vec{v}\|_{J_{0}(\Omega)}^{2}>0, \quad q=|L \vec{v}|^{2}>0, \quad q \leqslant \frac{J_{\ell}}{\rho}\|\vec{v}\|_{J_{0}(\Omega)}^{2}=\frac{J_{\ell}}{\rho} p .
$$

Therefore, we have

$$
\left\{\begin{array}{l}
(\beta-\varepsilon)(\beta-\gamma-\varepsilon) p-\frac{\rho}{J} \frac{(\beta-\varepsilon)^{3}(\beta-\gamma-\varepsilon)}{(\beta-\varepsilon)^{2}+\Lambda^{2}} q \\
\geqslant(\beta-\varepsilon)(\beta-\gamma-\varepsilon)\left[1-\frac{J_{\ell}}{J} \frac{(\beta-\varepsilon)^{3}}{(\beta-\varepsilon)^{2}+\Lambda^{2}}\right] p>0
\end{array}\right.
$$

and consequently

$$
(\mathcal{M}(\beta-\varepsilon) \vec{w}, \vec{w})_{J_{0}(\Omega)}>0
$$

\section{There exists a branch of positive real eigenvalues having infinity as a point of accumulation}

\subsection{Eigenvalues having infinity as a point of accumulation}

Setting $\widetilde{\lambda}=\lambda^{-1}$ and $\widetilde{\mathscr{L}}(\widetilde{\lambda})=\widetilde{\lambda} \mathscr{L}\left(\widetilde{\lambda}^{-1}\right)$, we have

$$
\widetilde{\mathscr{L}}(\widetilde{\lambda})=\nu \frac{\widetilde{\lambda}(1-\beta \widetilde{\lambda})}{1-\gamma \widetilde{\lambda}} I_{J_{0}(\Omega)}-A^{-1}+\frac{\rho}{J} \frac{1}{1+\Lambda^{2} \widetilde{\lambda}^{2}} B
$$

At first, we have

$$
\widetilde{\mathscr{L}}(0)=-A^{-1}+\frac{\rho}{J} B .
$$

This operator is compact like $A^{-1}$ and $B$; it is positive definite, because if $\vec{w}$ is a normalized vector of $J_{0}(\Omega)$, we have

$$
\left(\left(A^{-1}-\frac{\rho}{J} B\right) \vec{w}, \vec{w}\right)_{J_{0}(\Omega)}=p-\frac{\rho}{J} q>0, \text { since } p \geqslant \frac{\rho}{J_{\ell}} q .
$$

On the other hand, for the derivative of $\widetilde{\mathscr{L}}$, we have

$$
\widetilde{\mathscr{L}^{\prime}}(0)=\nu I_{J_{0}(\Omega)} \quad \text { strongly } \quad \text { positive. }
$$


Consequently [4], for each $\varepsilon>0$ sufficiently small there exists a branch of positive real eigenvalues $\widetilde{\lambda}_{n}^{\infty}$ set in $] 0, \varepsilon[$ that tend to zero as $n \rightarrow \infty$. The corresponding eigenelements form a Riesz basis in a subspace of $J_{0}(\Omega)$ having a finished defect.

Therefore, for our problem there is a denumerable infinity of eigenvalues $\lambda_{n}^{\infty}=\left(\widetilde{\lambda}_{n}^{\infty}\right)^{-1}$ having infinity as a point of accumulation.

\subsection{Asymptotic formula for $\lambda_{n}^{\infty}$}

Equation (7.5) can be written as

$$
\left(I_{J_{0}(\Omega)}-\nu^{-1} \frac{\lambda(\lambda-\gamma)}{\lambda-\beta} A^{-1}+\frac{\rho \nu^{-1}}{J} \frac{\lambda^{3}(\lambda-\gamma)}{(\lambda-\beta)\left(\lambda^{2}+\Lambda^{2}\right)} \mathcal{B}\right) \vec{w}=0 .
$$

Setting

$$
\frac{\lambda(\lambda-\gamma)}{\lambda-\beta}=\xi
$$

we find

$$
\lambda=\xi-\alpha+O\left(\frac{1}{\xi}\right)
$$

and the pencil

$$
I_{J_{0}(\Omega)}-\nu^{-1}\left(A^{-1}-\frac{\rho}{J} \mathcal{B}\right) \xi+O\left(\frac{1}{\xi}\right) \mathcal{B} \quad(\xi \text { large }) .
$$

We have applied the following theorem [4, pp. 71-72]:

Let the pencil

$$
L(\xi)=I+T-\xi Q+B(\xi)
$$

with $T$ compact, $Q$ compact, positive definite, $B(\xi)$ analytic outside the circle $|\xi| \leqslant R$ and turning into zero at infinity.

We have

$$
\lambda_{n}(L(\xi))=\frac{1}{\lambda_{n}(Q)}[1+o(1)], \quad n \rightarrow \infty .
$$

Here, the conditions are satified and we have

$$
\lambda_{n}^{\infty}=\frac{\nu}{\lambda_{n}\left(A^{-1}-\frac{\rho}{J} \mathcal{B}\right)}[1+o(1)], \quad n \rightarrow \infty .
$$

\section{There exists a branch of real eigenvalues $\lambda_{n}^{\beta}$ having $\beta$ as a point of accumulation}

\subsection{An operator pencil}

Setting

$$
\lambda=\lambda^{\prime \prime}+\beta \quad \text { and } \quad \widehat{\mathscr{L}}\left(\lambda^{\prime \prime}\right)=\frac{\mathscr{L}\left(\lambda^{\prime \prime}+\beta\right)}{\lambda^{\prime \prime}+\beta},
$$

we obtain the pencil

$$
\widehat{\mathscr{L}}\left(\lambda^{\prime \prime}\right)=\nu \frac{\lambda^{\prime \prime}}{\left(\lambda^{\prime \prime}+\beta\right)\left(\lambda^{\prime \prime}+\beta-\gamma\right)} I_{J_{0}(\Omega)}-A^{-1}+\frac{\rho}{J} \frac{\left(\lambda^{\prime \prime}+\beta\right)^{2}}{\left(\lambda^{\prime \prime}+\beta\right)^{2}+\Lambda^{2}} B .
$$


At first, we have

$$
\widehat{\mathscr{L}}(0)=-A^{-1}+\frac{\rho}{J} \frac{\beta^{2}}{\beta^{2}+\Lambda^{2}} B
$$

where $\widehat{\mathscr{L}}(0)$ is compact and negative definite. Indeed, if $\vec{w}$ is a normalized vector of $J_{0}(\Omega)$, we have

$$
\left(\left(A^{-1}-\frac{\rho}{J} \frac{\beta^{2}}{\beta^{2}+\Lambda^{2}} B\right) \vec{w}, \vec{w}\right)_{J_{0}(\Omega)}=p-\frac{\rho}{J} \frac{\beta^{2}}{\beta^{2}+\Lambda^{2}} q>p-\frac{\rho}{J} q>0 .
$$

On the other hand, we have

$$
\widehat{\mathscr{L}}^{\prime}(0)=\nu \frac{1}{\beta(\beta-\gamma)} I_{J_{0}(\Omega)}+\frac{\rho}{J} \frac{2 \beta \Lambda^{2}}{\left(\beta^{2}+\Lambda^{2}\right)^{2}} B
$$

obviously strongly positive.

On the other hand, we have seen in Section 9 that there are not real eigenvalues in $[\gamma, \beta]$.

Therefore, for each $\varepsilon>0$ sufficiently small, there exists a branch of positive real eigenvalues $\lambda_{n}^{\prime \prime}$ located in $] 0, \varepsilon[$ having zero as a point of accumulation. The eigenelements form a Riesz basis in some subspace of $J_{0}(\Omega)$ having a finite defect.

For our problem, there exists in $] \beta, \beta+\varepsilon[$ a denumerable infinity of real eigenvalues $\lambda_{n}^{\beta}=\lambda_{n}^{\prime \prime}+\beta$ having $\beta$ as an accumulation point.

\subsection{An asymptotic formula for $\lambda_{n}^{\beta}$}

After a somewhat long calculation that we omit, we have

$$
\lambda_{n}^{\beta}=\beta+\nu^{-1} \beta \alpha \lambda_{n}\left(A^{-1}-\frac{\rho}{J} \frac{\beta^{2}}{\beta^{2}+\Lambda^{2}} B\right)(1+o(1)), \quad n \rightarrow \infty .
$$

\section{Location of the possible nonreal eigenvalues}

Since $\lambda=0$ is not an eigenvalue, we consider the pencil

$$
\frac{\mathscr{L}(\lambda)}{\nu \lambda}=\left(\frac{\frac{\beta}{\gamma}}{\lambda}-\frac{\frac{\beta-\gamma}{\gamma}}{\lambda-\gamma}\right) I_{J_{0}(\Omega)}-\nu^{-1} A^{-1}+\frac{\rho \nu^{-1}}{J} \frac{\lambda^{2}}{\lambda^{2}+\Lambda^{2}} B .
$$

If $\lambda$ is a possible nonreal eigenvalue and $\vec{w}$ the corresponding normalized eigenvector, we have

$$
\left(\frac{\mathscr{L}(\lambda)}{\nu \lambda} \vec{w}, \vec{w}\right)_{J_{0}(\Omega)}=\frac{\frac{\beta}{\gamma}}{\lambda}-\frac{\frac{\beta-\gamma}{\gamma}}{\lambda-\gamma}-\nu^{-1} p+\frac{\rho \nu^{-1}}{J}\left(1-\frac{\Lambda^{2}}{\lambda^{2}+\Lambda^{2}}\right) q=0
$$

and then

$$
\operatorname{Im} \lambda\left[\frac{\frac{\beta}{\gamma}}{|\lambda|^{2}}-\frac{\frac{\beta-\gamma}{\gamma}}{|\lambda-\gamma|^{2}}-2 \frac{\rho \nu^{-1} \Lambda^{2}}{J} \operatorname{Re} \lambda \frac{q}{\left|\lambda^{2}+\Lambda^{2}\right|}\right]=0 .
$$

Consequently, we have

$$
\frac{\frac{\beta}{\gamma}}{|\lambda|^{2}}-\frac{\frac{\beta-\gamma}{\gamma}}{|\lambda-\gamma|^{2}} \geqslant 0
$$




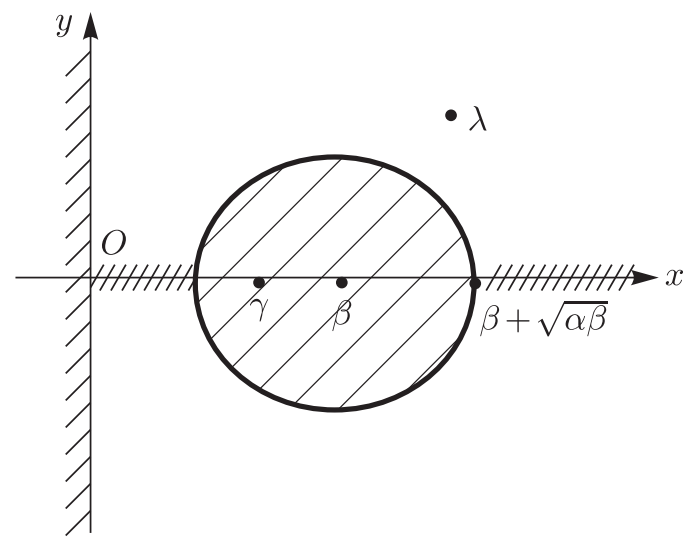

Fig. 2. Location of eigenvalues $\lambda_{n}$.

Setting $\lambda=x+i y$, we have

$$
(x-\beta)^{2}+y^{2} \geqslant \alpha \beta .
$$

Therefore, the possible nonreal eigenvalues are located outside the circle with center $\lambda=\beta$ and radius $\sqrt{\alpha \beta}$, which contains the point $\lambda=\gamma$.

In particular, $\beta$ cannot be a point of accumulation of nonreal eigenvalues.

\section{Acknowledgments}

The authors are grateful to the referee and the editorial board for some useful comments that improved the presentation of the manuscript.

\section{References}

[1] Essaouini, H., Elbahaoui, J., Elbakkali, L., and Capodanno, P., Mathematical Analysis of the Small Oscillations of a Heavy Heterogeneous Viscous Liquid in an Open Immovable Container, Eng. Math. Lett., 2014, vol. 2014, Art. ID 2, 17 pp.

[2] Essaouini, H., El Bakkali, L., and Capodanno, P., Analysis of the Small Oscillations of a Heavy Viscous Liquid in a Rigid Container Closed by an Elastic Membrane, Internat. J. Adv. Math., 2017, vol. 2017, no. 2, pp. 25-32.

[3] Essaouini, H., El Bakkali, L., and Capodanno, P., Mathematical Study of the Small Oscillations of Two Nonmixing Fluids, the Lower Inviscid, the Upper Viscoelastic, in an Open Container, J. Math. Fluid Mech., 2017, vol. 19, no. 4, pp. 645-657.

[4] Kopachevsky, N.D. and Krein, S. G., Operator Approach to Linear Problems of Hydrodynamics: Vol. 1. Self-Adjoint Problems for an Ideal Fluid, Oper. Theory Adv. Appl., vol. 128, Basel: Birkhäuser, 2001.

[5] Kopachevsky, N.D. and Krein, S. G., Operator Approach to Linear Problems of Hydrodynamics: Vol.2. Nonself-Adjoint Problems for Viscous Fluids, Oper. Theory Adv. Appl., vol. 146, Basel: Birkhäuser, 2003.

[6] Lions, J.L., Équations différentielles opérationnelles et problèmes aux limites, Grundlehren Math. Wiss., vol. 111, Berlin: Springer, 1961.

[7] Sánchez Hubert, J. and Sanchez-Palencia, E., Vibration and Coupling of Continuous Systems: Asymptotic Methods, Berlin: Springer, 1989. 